\title{
Modelos de Transporte de Contaminantes com Biodegradação na Subsuperfície
}

P.R.L. COUTO ${ }^{1}$, Departamento de Estudos Básicos e Instrumentais, Universidade Estadual do Sudoeste da Bahia, 15054-000 Campus de Itapetinga, Itapetinga, BA, Brasil

S.M.C. MALTA2, Coordenação de Matemática Aplicada e Computacional, LNCC/MCT, Av. Getúlio Vargas, 333, Quitandinha, 25651-070 Petrópolis, RJ, Brasil.

Resumo. Dois modelos matemáticos são propostos para o transporte reativo nãolinear de contaminantes na sub-superfície, o primeiro considerando o processo de biodegradação aeróbia de duas espécies de contaminantes, e o segundo sob condições aeróbia e anaeróbia de biodegradação de uma única espécie poluidora. Para resolver numericamente esses modelos é aplicada uma metodologia completamente acoplada (CA), no sentido que todos os operadores diferenciais de cada equação de transporte são discretizados e resolvidos simultaneamente como um sistema completo. Desta forma, são empregados os métodos SUPG (Streamline Upwind Petrov Galerkin), de elementos finitos, e Euler Implícito, de diferenças finitas, nas aproximações espaciais e temporais, respectivamente, e um algoritmo iterativo associado ao método de Newton na linearização e no desacoplamento dos sistemas de equações resultantes. Resultados numéricos são apresentados para demonstrar e ilustrar a eficiência das metodologias propostas quando aplicadas à modelagem de situações de interesse na contaminação de águas subterrâneas.

Palavras-chave. Modelagem matemática, métodos estabilizados de elementos finitos, transporte reativo não-linear.

\section{Introdução}

Modelos de transporte reativo não-lineares são bastante úteis para prever o comportamento de contaminantes na subsuperfície. Eles podem ser usados para planejar estratégias efetivas de bioremediação em regiões contaminadas, visando a proteção das reservas de águas subterrâneas. É usual modelar a biodegradação cineticamente, isto é, relacionando as taxas de utilização dos substratos, dos nutrientes e de crescimento microbiano às concentrações das substâncias envolvidas na reação,

\footnotetext{
${ }^{1}$ paularlcouto@gmail.com. A autora agradece ao $\mathrm{CNPq} / \mathrm{MCT}$ pelo apoio financeiro através do processo $\mathrm{n}^{\circ} 151796 / 2006-8$

${ }^{2}$ smcm@lncc.br. Parcialmente financiado pelo CNPq e FAPERJ/APQ1, processos 305747/2003-7 e E-26/171.356/2004
} 
tais como as dos próprios substratos e nutrientes [1, 4, 8]. Em geral, essas taxas são dependentes do que se denomina substrato limitante do crescimento, que é aquele de menor proporção e que é consumido primeiro [8]. Os modelos cinéticos são incluídos através de termos de fonte/sorvedouro nas equações de transporte das substâncias consideradas.

Neste trabalho dois modelos matemáticos são propostos para o transporte de contaminantes com biodegradação na subsuperfície, um levando-se em conta processos de biodegradação aeróbia de duas espécies de contaminantes e outro o transporte sob condições aeróbia e anaeróbia de biodegradação de uma única espécie poluidora [5]. Apesar destes modelos serem mais precisos do que aqueles usualmente estudados na literatura, em que apenas processos lineares estão envolvidos, suas não linearidades e os acoplamentos presentes dificultam a obtenção de soluções analíticas para esta classe de problemas. Portanto, aproximações numéricas devem ser aplicadas para resolver aproximadamente os sistemas de equações resultantes. Assim, uma metodologia numérica completamente acoplada (CA) é desenvolvida e empregada na simulação dos modelos em questão. Ela é denominada completamente acoplada no sentido que todos os operadores diferenciais de cada equação de transporte são discretizados e resolvidos simultaneamente como um sistema completo. A metodologia é seqüencialmente implícita, pois as equações são resolvidas, uma após a outra, de forma seqüencial e dependendo das outras variáveis do sistema. Os métodos de elementos finitos estabilizados SUPG (Streamline Upwind Petrov Galerkin) e de diferenças finitas Euler Implícito são usados e aplica-se um algoritmo iterativo associado ao método de Newton para a linearização e para o desacoplamento do sistema de equações.

Este trabalho está organizado na seguinte estrutura. Na Seção 2 um modelo geral não-linear descrevendo o transporte de contaminantes em um meio poroso subsuperficial é introduzido. Dessa forma, define-se os problemas de interesse e seus resultados numéricos como simples extensões, nas Seções 4 e 5, respectivamente. A metodologia numérica desenvolvida para resolvê-los encontra-se na Seção 3. Por último, na Seção 6, são feitas algumas considerações e conclusões.

\section{O Modelo Geral}

Com o objetivo de definir claramente a metodologia numérica e a modelagem matemática dos problemas propostos, apresenta-se aqui um modelo geral de equações diferenciais não-lineares acopladas que descreve o transporte de um doador de elétrons (contaminante) $\left(C_{1}\right)$, de um aceptor de elétrons $\left(C_{2}\right)$ e do crescimento de uma biomassa bacteriana $\left(b_{1}\right)$ na sub-superfície. Portanto, as equações de balanço de massa para o doador de elétrons na fase fluida $\left(C_{1}\right)$, para o aceptor de elétrons $\left(C_{2}\right)$ e para a biomassa $\left(b_{1}\right)$ são dadas por

$$
\begin{aligned}
& \frac{\partial C_{1}}{\partial t}+V \frac{\partial C_{1}}{\partial x}-D \frac{\partial^{2} C_{1}}{\partial x^{2}}+R_{1}\left(C_{1}, C_{2}, b_{1}\right)=0 \\
& \frac{\partial C_{2}}{\partial t}+V \frac{\partial C_{2}}{\partial x}-D \frac{\partial^{2} C_{2}}{\partial x^{2}}+R_{2}\left(C_{1}, C_{2}, b_{1}\right)=0
\end{aligned}
$$




$$
\frac{\partial b_{1}}{\partial t}=R_{3}\left(C_{1}, C_{2}, b_{1}\right)
$$

para todo $x \in(0, L)$ e $t>0$. Os processos de biodegradação são introduzidos pelas cinéticas de Monod de taxa mínima, definidas pelas funções não-lineares $R_{i}(\cdot, \cdot, \cdot)$, $i=1,2,3$, apresentadas nas Seções 4 e 5 . Para resolver numericamente o modelo não-linear proposto acima é necessário ainda definir condições de contorno e iniciais apropriadas para a região de estudo considerada, $\Omega=[0, L]$. Para o contorno direito $(x=L)$, assume-se que a condição de derivada nula é válida e para o contorno esquerdo $(x=0)$ estabelecem-se condições de Dirichlet, isto é,

$$
\frac{\partial C_{i}(L, t)}{\partial x}=0, \quad C_{i}(0, t)=\bar{C}_{i}, \quad i=1,2 .
$$

Além disso, condições iniciais são dadas por

$$
C_{i}(x, 0)=C_{i}^{0}, \quad b_{1}(x, 0)=b_{1}^{0} \quad i=1,2 .
$$

Em (2.4)-(2.5), $\bar{C}_{i}, C_{i}^{0}, i=1,2$, e $b_{1}^{0}$ são constantes conhecidas. Portanto, o sistema (2.1)-(2.3), juntamente com as condições de contorno (2.4) e com as condições iniciais (2.5) constitui o modelo padrão para o problema de determinar numericamente as variáveis $C_{1}, C_{2}$ e $b_{1}$. O leitor interessado por um modelo ainda mais geral, que analise os efeitos da combinação dos processos de sorção e de biodegração sobre o transporte de contaminantes poderá consultar [5].

\section{A Metodologia Completamente Acoplada (CA)}

A seguir, apresenta-se a metodologia numérica completamente acoplada (CA) empregada na resolução do modelo padrão (2.1)-(2.5). Seja $0=t^{0}<t^{1}<t^{2}<\ldots<$ $t^{N}=T$ uma partição de $I \equiv[0, T]$. Considera-se que o tempo é uniformemente dividido e o passo de tempo é dado por $\Delta t=t^{n}-t^{n-1}, n=1, \ldots, N$, com $N=T / \Delta t$. Então, aplica-se o método Euler Implícito de diferenças finitas na aproximação das derivadas temporais. Denotando por $C_{i}^{n}=C_{i}\left(x, t^{n}\right), i=1,2$ e $b_{1}^{n}=b_{1}\left(x, t^{n}\right)$, segue que

$$
\begin{gathered}
\mathcal{R}_{1}\left(C_{1}^{n}\right)=C_{1}^{n}-C_{1}^{n-1}+\Delta t\left(V \frac{\partial C_{1}^{n}}{\partial x}-D \frac{\partial^{2} C_{1}^{n}}{\partial x^{2}}+R_{1}\left(C_{1}^{n}, C_{2}^{n}, b_{1}^{n}\right)\right)=0, \\
\mathcal{R}_{2}\left(C_{2}^{n}\right)=C_{2}^{n}-C_{2}^{n-1}+\Delta t\left(V \frac{\partial C_{2}^{n}}{\partial x}-D \frac{\partial^{2} C_{2}^{n}}{\partial x^{2}}+R_{2}\left(C_{1}^{n}, C_{2}^{n}, b_{1}^{n}\right)\right)=0, \\
b_{1}^{n}-b_{1}^{n-1}=\Delta t R_{3}\left(C_{1}^{n}, C_{2}^{n}, b_{1}^{n}\right),
\end{gathered}
$$

é o sistema discretizado no tempo associado ao problema contínuo (2.1)-(2.3), onde $\mathcal{R}_{i}\left(C_{i}^{n}\right), i=1,2$, são os resíduos das equações de transporte (2.1) e (2.2), respectivamente.

A discretização completa é obtida através da aplicação do método de elementos finitos SUPG (Streamline Upwind Petrov Galerkin) [3] nas equações (3.1) e (3.2). 
Este método foi escolhido já que problemas de transporte com biodegradação em meios porosos são considerados predominantemente advectivos [1, 2, 8] e o método de Galerkin usual apresenta oscilações não físicas neste caso [3]. Seja o domínio $\Omega$ particionado em $N_{e}$ elementos com tamanho $h=\Delta x=L / N_{e}$ (malha uniforme). Cada elemento $\Omega_{e}$ é tal que $\bar{\Omega}=\bigcup_{e=1}^{N_{e}} \bar{\Omega}_{e}$ com $\bigcap_{e=1}^{N_{e}} \Omega_{e}=\emptyset$. Logo, os problemas de transporte (2.1) e (2.2), totalmente discretos, são definidos por:

(i) dados $C_{1}^{n-1, h}, C_{2}^{n, h}$ e $b_{1}^{n, h}$ encontrar, $\forall n=1, \ldots, N, C_{1}^{n, h} \in V_{1}^{h}$ satisfazendo

$$
\int_{\Omega} \mathcal{R}_{1}\left(C_{1}^{n, h}\right) w^{h} d x+\sum_{e=1}^{N_{e}} \int_{\Omega_{e}} \mathcal{R}_{1}\left(C_{1}^{n, h}\right) \tau V \frac{\partial w^{h}}{\partial x} d x=0 \quad \forall w^{h} \in W^{h}
$$

(ii) dados $C_{2}^{n-1, h}, C_{1}^{n, h}$ e $b_{1}^{n, h}$, encontrar, $\forall n=1, \ldots, N, C_{2}^{n, h} \in V_{2}^{h}$ satisfazendo

$$
\int_{\Omega} \mathcal{R}_{2}\left(C_{2}^{n, h}\right) w^{h} d x+\sum_{e=1}^{N_{e}} \int_{\Omega_{e}} \mathcal{R}_{2}\left(C_{2}^{n, h}\right) \tau V \frac{\partial w^{h}}{\partial x} d x=0 \quad \forall w^{h} \in W^{h} .
$$

O sobreescrito $h$ refere-se à solução na malha de elementos finitos. O parâmetro de upwind $\tau$ é definido por $\tau=h \xi(P e) / 2|V|[3]$, onde $\xi(P e)=\operatorname{coth}(P e)-(1 / P e) \operatorname{com}$ $P e=|V| h / 2 D$ o número de Peclet de malha (adimensional), que avalia a relação entre os termos advectivos e dispersivos. $V_{i}^{h}$ e $W^{h}$ são as contrapartidas finitas dos espaços $V_{i}=\left\{c_{i}(x) \in H^{1}(\Omega) ; c_{i}(0)=\bar{C}_{i}\right\}, i=1,2$ e $W=\left\{w(x) \in H^{1}(\Omega) ; w(0)=\right.$ $0\}$. Neste trabalho, utilizam-se apenas elementos lineares. As não linearidades e o acoplamento são inseridos nas equações totalmente discretizadas (3.4) e (3.5) através dos termos $R_{i}(\cdot, \cdot, \cdot), i=1,2,3$, dependendo das cinéticas escolhidas.

Daqui em diante, para simplificar a notação, substitui-se $C_{i}^{n, h}$ por $c_{i}^{n}$ e $b_{1}^{n, h}$ por $b_{1}^{n}$. Para tratar as não linearidades e o acoplamento propõe-se um algoritmo baseado no método de Newton [6,9]. Primeiramente, as funções não lineares são substituídas (numa certa vizinhança) por sua aproximação linear, obtida tomando os primeiros termos do seu desenvolvimento em série de Taylor. Em seguida, substitui-se esta aproximação nas equações (3.4) e (3.5) e determinam-se as soluções para as equações linearizadas. Então o processo é repetido sistematicamente até que seja atingido o grau de aproximação desejado. Para descrever este processo iterativo, denota-se o índice de iteração por $k$. As funções $R_{i}(\cdot, \cdot, \cdot), i=1,2,3$, no nível de iteração $k$, são então aproximadas por curvas tangentes em $c_{i}^{n, k-1}$. Substituindo-se essas aproximações lineares nos termos $\mathcal{R}_{1}(\cdot)$ e $\mathcal{R}_{2}(\cdot)$ de (3.1)e (3.2), obtém-se

$$
\begin{aligned}
\mathcal{R}_{1}\left(c_{1}^{n, k}\right)= & c_{1}^{n, k}-c_{1}^{n-1}+\Delta t\left[\frac{\partial c_{1}^{n, k}}{\partial x}-D \frac{\partial^{2} c_{1}^{n, k}}{\partial x^{2}}+\left(R_{1}\left(c_{1}^{n, k-1}, c_{2}^{n, k-1}, b_{1}^{n, k-1}\right)\right.\right. \\
& \left.\left.+\frac{d R_{1}\left(c_{1}^{n, k-1}, c_{2}^{n, k-1}, b_{1}^{n, k-1}\right)}{d C_{1}}\left(c_{1}^{n, k}-c_{1}^{n, k-1}\right)\right)\right]
\end{aligned}
$$


e

$$
\begin{aligned}
\mathcal{R}_{2}\left(c_{2}^{n, k}\right)= & c_{2}^{n, k}-c_{2}^{n-1}+\Delta t\left[V \frac{\partial c_{2}^{n, k}}{\partial x}-D \frac{\partial^{2} c_{2}^{n, k}}{\partial x^{2}}+\left(R_{2}\left(c_{1}^{n, k-1}, c_{2}^{n, k-1}, b_{1}^{n, k-1}\right)\right.\right. \\
& \left.\left.+\frac{d R_{2}\left(c_{1}^{n, k-1}, c_{2}^{n, k-1}, b_{1}^{n, k-1}\right)}{d C_{2}}\left(c_{1}^{n, k}-c_{1}^{n, k-1}\right)\right)\right]
\end{aligned}
$$

Combinam-se então as formulações dos problemas de transporte totalmente discretas (3.4)-(3.5) com as aproximações linearizadas (3.6)-(3.7), considerando-se a aproximação por diferenças finitas do problema de crescimento bacteriano, equação (3.3), em um esquema iterativo do tipo Newton. Portanto, é construído um algoritmo iterativo para a solução do problema padrão (2.1)-(2.5) em cada tempo $t^{n}$, $n=1, \ldots, N[5]$. Neste algoritmo, a solução em cada tempo $t^{n}$ é encontrada quando as soluções $x^{k}$ e $x^{k-1}$, obtidas em duas iterações consecutivas, estão suficientemente próximas, de modo que $\left\|x^{k}-x^{k-1}\right\|=$ má $_{p=1}^{n n o d e}\left\|x_{p}^{k}-x_{p}^{k-1}\right\|<$ tol, onde tol é a tolerância pré-estabelecida e $k_{\max }$ é o número máximo de iterações em cada passo de tempo onde o algoritmo é aplicado.

Observa-se que a metodologia proposta é seqüencialmente implícita, pois as equações são resolvidas, uma após a outra, de forma seqüencial e dependendo das outras variáveis do sistema.

A seguir, a partir do modelo geral (2.1)-(2.3), serão apresentados dois problemas de transporte de contaminantes na sub-superfície considerando processos de biodegradação aeróbia e anaeróbia.

\section{Primeiro Modelo}

Descreve-se nessa seção a modelagem matemática para um problema de transporte com biodegradação aeróbia de duas espécies de contaminantes na sub-superfície, ilustrado por um cenário de contaminação onde dois doadores de elétrons (contaminantes) são degradados pela presença de uma biomassa aeróbia, através do mesmo sistema de enzimas. Ou seja, acontecerá competição entre os doadores de elétrons pelo mesmo sítio enzimático, responsável pela reação. Desta forma, seja $C_{1}$ a concentração do primeiro doador (doador 1) de elétrons na fase fluida, que provoca inibição no segundo doador (doador 2) presente, representado pela concentração $C_{3}$ e, finalmente, seja $C_{2}$ a concentração do aceptor de elétrons e $b_{1}$ a quantidade de biomassa. Portanto, o sistema de transporte reativo não-linear com biodegradação aeróbia para as varáveis em estudo é descrito pelo modelo geral (2.1)-(2.3) acrescido de mais uma equação de transporte, para $C_{3}$, ou seja,

$$
\frac{\partial C_{3}}{\partial t}+V \frac{\partial C_{3}}{\partial x}-D \frac{\partial^{2} C_{3}}{\partial x^{2}}+R_{4}\left(C_{1}, C_{2}, C_{3}, b_{1}\right)=0 .
$$

A competição pelo mesmo sítio de enzimas, mencionada acima, pode ser modelada introduzindo-se um fator de inibição competitiva $I_{b}$ no modelo cinético do doador que seria naturalmente degradado pela biomassa se o outro doador não estivesse 
presente. Supõe-se que a degradação de um outro doador presente no meio provoca o crescimento da biomassa e aumenta o consumo do aceptor de elétrons. Além disso, considera-se que o substrato limitante do crescimento são os dois doadores juntos ou o aceptor, ou seja, como existem dois doadores de elétrons, o aceptor será limitante quando sua concentração for menor que a concentração de qualquer um dos doadores. Para representar claramente essas hipóteses e definir totalmente o modelo de transporte a ser estudado, apresenta-se, a seguir, as expressões cinéticas para todas as espécies envolvidas $\left(R_{i}\left(C_{1}, C_{2}, C_{3}, b_{1}\right)\right), i=1, \ldots, 4$, dadas por cinéticas de Monod de taxa mínima [8]. Deste modo, tem-se

$$
\begin{gathered}
R_{1}\left(C_{1}, C_{2}, C_{3}, b_{1}\right)=(1-\gamma) K_{1}\left(C_{1}\right)+\gamma \frac{Y_{C_{2}}}{Y_{C_{1}}} K_{2}\left(C_{2}\right), \\
R_{2}\left(C_{1}, C_{2}, C_{3}, b_{1}\right)=\gamma K_{2}\left(C_{2}\right)+(1-\gamma)\left[\frac{Y_{C_{1}}}{Y_{C_{2}}} K_{1}\left(C_{1}\right)+\frac{Y_{C_{3}}}{Y_{C_{2}}} K_{3}\left(C_{3}\right)\right], \\
R_{3}\left(C_{1}, C_{2}, C_{3}, b_{1}\right)=(1-\gamma)\left[Y_{C_{1}} K_{1}\left(C_{1}\right)+Y_{C_{3}} K_{3}\left(C_{3}\right)+\gamma Y_{C_{2}} K_{2}\left(C_{2}\right)-m b_{1},\right. \\
R_{4}\left(C_{1}, C_{2}, C_{3}, b_{1}\right)=(1-\gamma) K_{3}\left(C_{3}\right)+\gamma \frac{Y_{C_{2}}}{Y_{C_{3}}} \tilde{K}_{2}\left(C_{2}\right), \\
K_{j}\left(C_{j}\right)=\frac{V_{m}^{1}\left(C_{j}\right) b_{1} C_{j}}{I_{b}\left(K_{h}^{1}\left(C_{j}\right)+C_{j}\right)}, j=1,2, \quad K_{3}\left(C_{3}\right)=\frac{V_{m}^{1}\left(C_{3}\right) b_{1} C_{3}}{I_{b}\left(K_{h}^{1}\left(C_{3}\right) I_{c}+C_{3}\right)}, \\
\gamma=\gamma\left(C_{2}\left(C_{2}\right)=\frac{V_{m}^{1}\left(C_{2}\right) b_{1} C_{2}}{I_{b}\left(K_{h}^{1}\left(C_{2}\right) I_{c}+C_{2}\right)}, \quad I_{c}=1+\frac{C_{1}}{K_{c}}, \quad I_{b}=1+\frac{b_{1}}{K_{b}},\right. \\
\left\{\begin{array}{l}
1 \mathrm{se} C_{2} \leq C_{1} \text { ou } C_{2} \leq C_{3} \text { ou }\left(C_{2}>C_{1} \mathrm{e} C_{2}>C_{3} \mathrm{e} C_{1}+C_{3}>C_{2}\right), \\
0 \mathrm{se} C_{2}>C_{1} \mathrm{e} C_{2}>C_{3} \mathrm{e} C_{1}+C_{3} \leq C_{2} .
\end{array}\right.
\end{gathered}
$$

É importante destacar que o doador 1 não sofre efeito direto da competição com o doador 2, mas ele influencia diretamente o doador 2. Portanto, considera-se que o doador 1 é de certa forma "mais forte" do que o doador 2. Além disso, deve-se observar que o fator de inibição competitiva $I_{c}$ é inserido no termo de reação do doador 2, equação (4.5), tanto no caso em que o aceptor de elétrons é limitante $(\gamma=1)$ quanto na situação onde os doadores são limitantes $(\gamma=0)$. Simulações foram realizadas para este primeiro modelo usando os parâmetros de discretização dados Tabela 1 , onde os valores para o campo de velocidades $(V)$ e o coeficiente de difusão $(D)$ foram adotados de acordo com o trabalho de Krinded e Celia [8].

Tabela 1: Parâmetros de discretização.

\begin{tabular}{c|c|c|c|c|c|c}
\hline$\Delta x$ & $\Delta t$ & $V$ & $D$ & $P e$ & $N e$ & nnode \\
\hline \hline $0,5 m$ & 0,5 dia & 1,0 mdia $^{-1}$ & $0,2 m^{2}$ dia $^{-1}$ & 1,25 & 200 & 201 \\
\hline
\end{tabular}

Além disso, na Tabela 2 apresenta-se os parâmetros cinéticos considerados [1, $2,8]$ para o cálculo das expressões $R_{i}(\cdot, \cdot, \cdot),=1, \ldots, 4$, definidas em (4.2)-(4.5). 
Tabela 2: Parâmetros cinéticos.

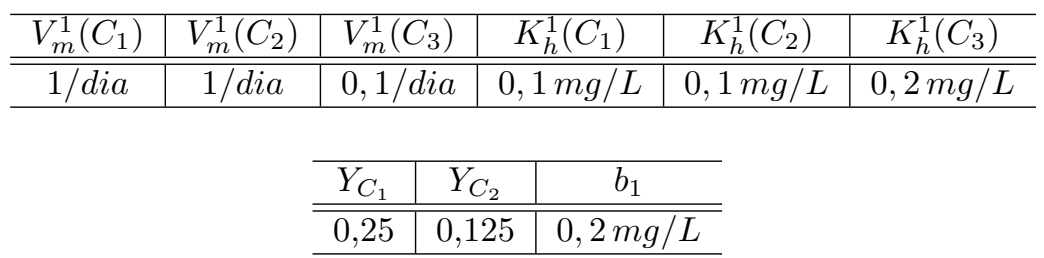

Finalmente, as constantes de inibição competitiva e de acúmulo de biomassa usadas são [8]: $K_{c}=0,001 m g L^{-1}$ e $K_{b}=1,0 m g L^{-1}$ e $Y_{C_{3}}=Y_{C_{1}}$.

As Figuras 1-4 mostram a evolução do comportamento das quatro espécies envolvidas para 34 (esquerda) e 68 (direita) dias, com variações nas condições no contorno esquerdo para $C_{1}, C_{2}$ e $C_{3}$ e nas condições iniciais para $C_{2}$. Mantendo-se as condições iniciais para $C_{1}(t, x)$ e $C_{3}(t, x)$ sempre nulas, ou seja, $C_{1}(0, x)=0$ e $C_{3}(0, x)=0$ variar-se-á somente a condição inicial para $C_{2}(t, x)$ nos casos simulados. Deste modo, o doador $1\left(C_{1}\right)$ está representado pela linha tracejada, o aceptor de elétrons $\left(C_{2}\right)$ pela linha contínua, a biomassa $\left(b_{1}\right)$ pela linha pontilhada e o doador $2\left(C_{3}\right)$ pela linha traço-ponto.
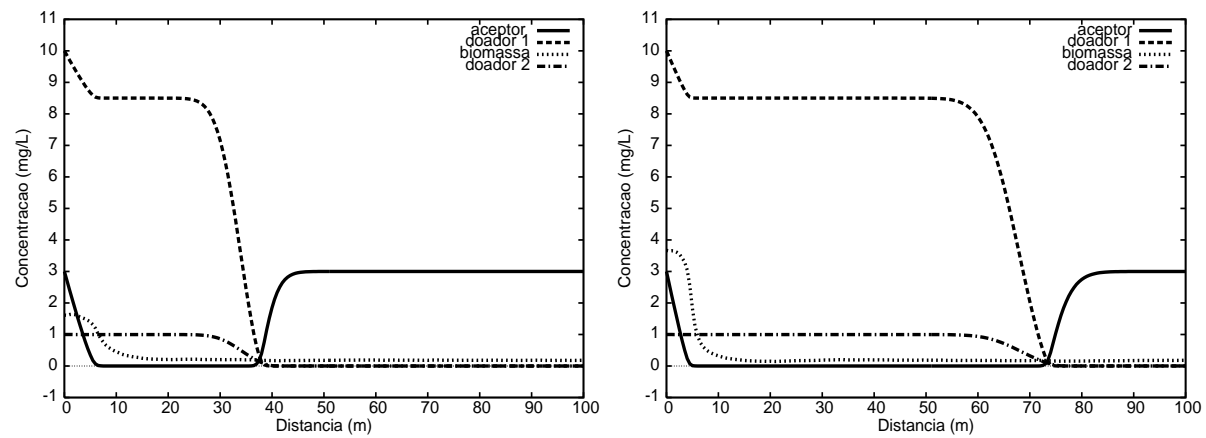

Figura 1: Perfis das concentrações para o $1^{\circ}$ modelo em 34 dias (esquerda) e 68 dias (direita). $C_{1}(0, t)=10 m g L^{-1}, C_{2}(0, t)=3 m g L^{-1}, C_{3}(0, t)=1 m g L^{-1}, C_{2}(x, 0)=$ $3 m g L^{-1}$.

Observa-se nas Figuras 1 e 2 que a taxa de biodegradação do doador 2 na presença do doador 1 é reduzida e quase não se percebe, por causa da introdução do fator de inibição competitiva em sua cinética. Na Figura 1, nota-se que, mesmo onde o doador 1 está ausente, a biodegradação do doador 2 não aparece, pois todo aceptor disponível foi usado na degradação do doador 1. Quando a concentração inicial do doador 1 é pequena, como na Figura 2, a concentração do aceptor é suficiente para garantir uma taxa maior de biodegradação do doador 2 nos lugares onde o doador 1 foi completamente biodegradado. Na Figura 3 aumentou-se o nível de aceptor e foram injetadas concentrações menores de doadores. Pode-se ver então que embora haja a inibição do doador 2 durante a degradação do doador 

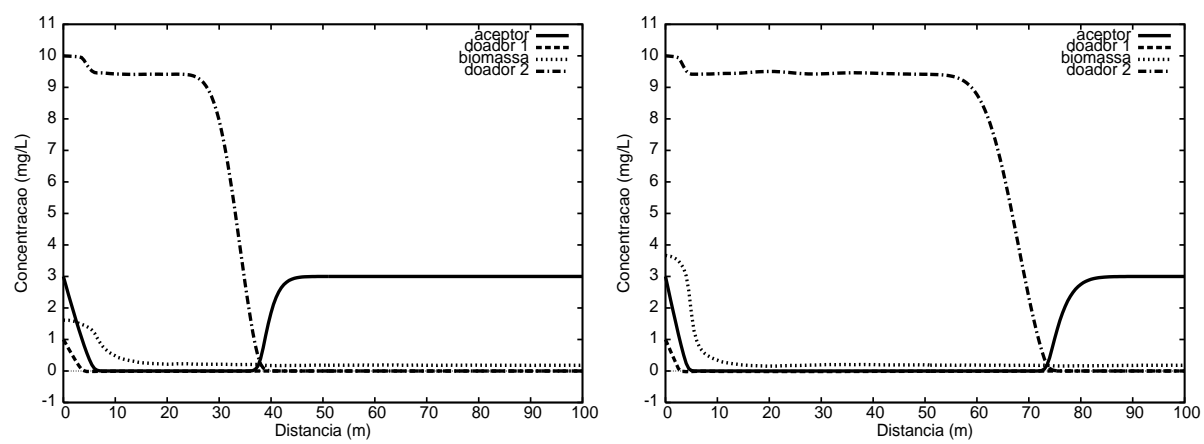

Figura 2: Perfis das concentrações para o $1^{\circ}$ modelo em 34 dias (esquerda) e 68 dias (direita). $C_{1}(0, t)=1 m g L^{-1}, C_{2}(0, t)=3 m g L^{-1}, C_{3}(0, t)=10 m g L^{-1}$, $C_{2}(x, 0)=3 m g L^{-1}$.
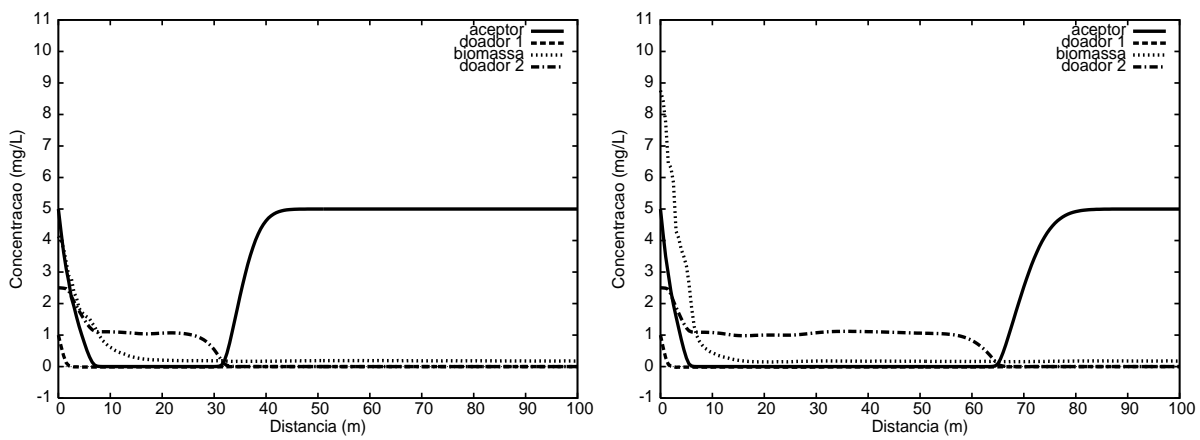

Figura 3: Perfis de concentração para o $1^{\circ}$ modelo em 34 dias (esquerda) e 68 dias (direita). $C_{1}(0, t)=1 m g L^{-1}, C_{2}(0, t)=5 m g L^{-1}, C_{3}(0, t)=2,5 m g L^{-1}$, $C_{2}(x, 0)=5 m g L^{-1}$.
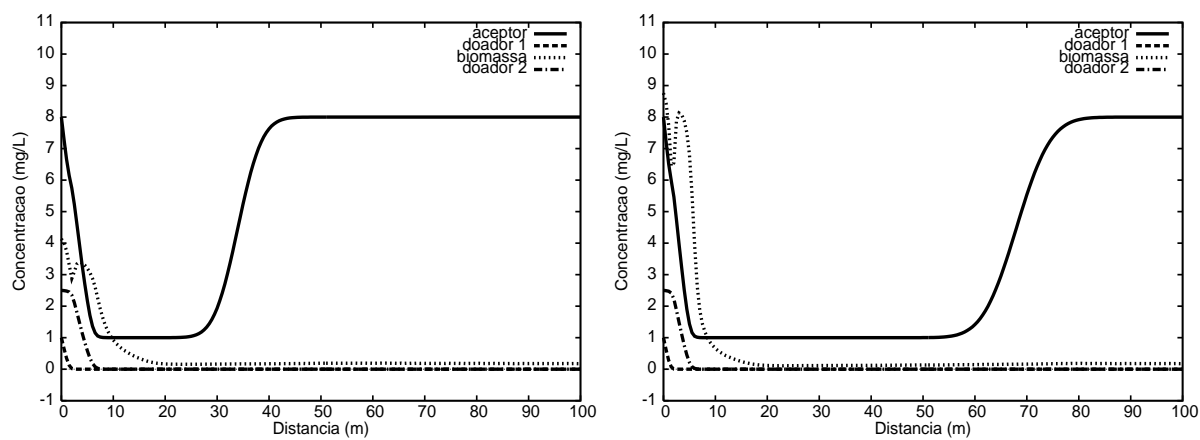

Figura 4: Perfis de concentração para o $1^{\circ}$ modelo em 34 dias (esquerda) e 68 dias (direita). $C_{1}(0, t)=1 m g L^{-1}, C_{2}(0, t)=8 m g L^{-1}, C_{3}(0, t)=2,5 m g L^{-1}$, $C_{2}(x, 0)=8 m g L^{-1}$. 
1, a quantidade de aceptor é suficiente para degradar parte do doador 2, antes que este atinja regiões mais distantes da fonte de contaminação. Nota-se que o crescimento bacteriano foi acentuado nesta situação. Quando o oxigênio/aceptor $\left(C_{2}(0, t)=8 m g L^{-1}\right)$ é abundante, Figura 4 , os dois doadores são completamente degradados perto da fonte de contaminação e sua plumas não são propagadas no domínio, conforme tem sido ilustrado na literatura [8].

\section{Segundo Modelo}

Neste segundo modelo estuda-se o transporte de um contaminante com biodegradação aeróbia e anaeróbia. Apesar da rota metabólica aeróbia ser preferencialmente utilizada pelos microorganismos subsuperficiais, na ausência do oxigênio nota-se que alguns substratos são degradados devido à presença de microorganismos anaeróbios facultativos ou obrigatórios, que utilizam outro tipo de aceptor de elétrons para a degradação. Neste caso, simula-se aqui um cenário de contaminação devido ao transporte e a degradação de um doador de elétrons (contaminante, $C_{1}$ ), que é degradado por dois grupos de biomassa, um aeróbio e outro anaeróbio. $\mathrm{O}$ grupo aeróbio (biomassa 1, $b_{1}$ ), como no modelo anterior, utiliza como aceptor de elétrons o oxigênio (aceptor 1, $C_{2}$ ) e considera-se que o grupo anaeróbio (biomassa $\left.2, b_{2}\right)$ utiliza, por exemplo, o nitrato (aceptor $2, C_{3}$ ). Supõe-se ainda que a presença do aceptor 1 inibe o metabolismo anaeróbio e este comportamento pode ser modelado através de um fator $I_{n}$ de inibição não competitiva, onde a substância inibidora é o oxigênio. Portanto, o sistema de equações que descreve o cenário de contaminação em estudo é composto de cinco equações, a saber: as quatro equações do primeiro modelo, correspondentes as variáveis $C_{1}, C_{2}, b_{1}$ e $C_{3}$, acrescidas de uma nova equação de crescimento de biomassa para a variável $b_{2}$, ou seja,

$$
\frac{\partial b_{2}}{\partial t}=R_{5}\left(C_{1}, C_{2}, C_{3}, b_{1}, b_{2}\right) .
$$

As expressões cinéticas $\left(R_{i}\left(C_{1}, C_{2}, C_{3}, b_{1}, b_{2}\right), i=1, \ldots, 5\right)$ são agora definidas através de cinéticas de Monod de taxa mínina escritas em função das cinco variáveis envolvidas. Desta forma, tem-se

$$
\begin{gathered}
R_{1}\left(C_{1}, C_{2}, C_{3}, b_{1}, b_{2}\right)=\left(1-\gamma_{1}\right) K_{1}^{1}\left(C_{1}\right)+\left(1-\gamma_{2}\right) K_{1}^{2}\left(C_{1}\right) \\
+\gamma_{1} \frac{Y_{C_{2}}^{1}}{Y_{C_{1}}^{1}} K_{2}^{1}\left(C_{2}\right)+\gamma_{2} \frac{Y_{C_{3}}^{2}}{Y_{C_{1}}^{2}} K_{3}^{2}\left(C_{3}\right), \\
R_{2}\left(C_{1}, C_{2}, C_{3}, b_{1}, b_{2}\right)=\gamma_{1} K_{2}^{1}\left(C_{2}\right)+\left(1-\gamma_{1}\right) \frac{Y_{C_{1}}^{1}}{Y_{C_{2}}^{1}} K_{1}^{1}\left(C_{1}\right), \\
R_{3}\left(C_{1}, C_{2}, C_{3}, b_{1}, b_{2}\right)=\left(1-\gamma_{1}\right) Y_{C_{1}}^{1} K_{1}^{1}\left(C_{1}\right)+\gamma_{1} Y_{C_{2}}^{1} K_{2}^{1}\left(C_{2}\right)-m_{1} b_{1}, \\
R_{4}\left(C_{1}, C_{2}, C_{3}, b_{1}, b_{2}\right)=\gamma_{2} K_{3}^{2}\left(C_{3}\right)+\left(1-\gamma_{2}\right) \frac{Y_{C_{1}}^{2}}{Y_{C_{3}}^{2}} K_{1}^{2}\left(C_{1}\right), \\
R_{5}\left(C_{1}, C_{2}, C_{3}, b_{1}, b_{2}\right)=\left(1-\gamma_{2}\right) Y_{C_{1}}^{2} K_{1}^{2}\left(C_{1}\right)+\gamma_{2} Y_{C_{3}}^{2} K_{3}^{2}\left(C_{3}\right)-m_{2} b_{2},
\end{gathered}
$$




$$
\begin{gathered}
K_{j}^{1}\left(C_{j}\right)=\frac{V_{m}^{1}\left(C_{j}\right) b_{1} C_{j}}{I_{b_{1}}\left(K_{h}^{1}\left(C_{j}\right)+C_{j}\right)}, j=1,2, \\
K_{i}^{2}\left(C_{i}\right)=\frac{V_{m}^{2}\left(C_{i}\right) b_{2} C_{i}}{I_{b_{2}} I_{n}\left(K_{h}^{2}\left(C_{i}\right)+C_{i}\right)}, i=1,3, \\
I_{n}=1+\frac{C_{2}}{K_{n}}, \gamma_{1}=\gamma_{1}\left(C_{1}, C_{2}\right)=\left\{\begin{array}{l}
\left.0 \text { se } C_{1}<C_{2} \text { (o doador limita } b_{1}\right), \\
\left.1 \text { se } C_{2} \leq C_{1} \text { (o aceptor } 1 \text { limita } b_{1}\right),
\end{array}\right. \\
\gamma_{2}=\gamma_{2}\left(C_{1}, C_{3}\right)=\left\{\begin{array}{l}
\left.0 \text { se } C_{1}<C_{3} \text { (o doador limita } b_{2}\right), \\
\left.1 \text { se } C_{3} \leq C_{1} \text { (o aceptor } 2 \text { limita } b_{2}\right) .
\end{array}\right.
\end{gathered}
$$

Nas equações (5.4)-(5.7), a constante $m_{i}$ e o fator $I_{b_{i}}$ são, respectivamente, o coeficiente de decaimento e o fator de inibição por biomassa relativos a $b_{i}, i=1,2$. A constante de inibição correspondente a $I_{b_{i}}$ é $K_{b_{i}}$. As constantes $Y_{C_{j}}^{i}, j=1,2,3$, nas equações (5.2)-(5.6) são os coeficientes de produção da biomassa $i, i=1,2$. Os parâmetros de discretização empregados aqui são os mesmos da Tabela 1 da seção anterior; os demais parâmetros, condições iniciais e de contorno são dados na Tabela $3[1,2,8]$.

Tabela 3: Parâmetros para o segundo modelo.

\begin{tabular}{cc|cc|cc}
\hline Parâmetro & Valor & Parâmetro & Valor & C.I.C. $^{*}$ & Valor \\
\hline \hline$V_{m}^{1}\left(C_{1}\right)$ & $1,0 \mathrm{dia}^{-1}$ & $Y_{C_{1}}^{1}$ & 0,250 & $C_{1}(0, t)$ & $10,0 \mathrm{mgL}^{-1}$ \\
$V_{m}^{2}\left(C_{1}\right)$ & $1,0 \mathrm{dia}^{-1}$ & $Y_{C_{1}}^{2}$ & 0,200 & $C_{2}(0, t)$ & $03,0 \mathrm{mgL}^{-1}$ \\
$V_{m}^{1}\left(C_{2}\right)$ & $1,0 \mathrm{dia}^{-1}$ & $Y_{C_{2}}^{1}$ & 0,125 & $C_{3}(0, t)$ & $02,0 \mathrm{mgL}^{-1}$ \\
$V_{m}^{2}\left(C_{3}\right)$ & $1,0 \mathrm{dia}^{-1}$ & $Y_{C_{3}}^{2}$ & 0,100 & $C_{1}(x, 0)$ & $00,0 \mathrm{mgL}^{-1}$ \\
$K_{h}^{1}\left(C_{1}\right)$ & $0,1 \mathrm{mgL}^{-1}$ & $m_{1}$ & 0,010 & $C_{2}(x, 0)$ & $03,0 \mathrm{mgL}^{-1}$ \\
$K_{h}^{2}\left(C_{1}\right)$ & $0,1 \mathrm{mgL}^{-1}$ & $m_{2}$ & 0,010 & $C_{3}(x, 0)$ & $00,0 \mathrm{mgL}^{-1}$ \\
$K_{h}^{1}\left(C_{2}\right)$ & $0,1 \mathrm{mgL}^{-1}$ & $K_{n}$ & 0,100 & $b_{1}(x, 0)$ & $00,1 \mathrm{mgL}^{-1}$ \\
$K_{h}^{2}\left(C_{3}\right)$ & $0,1 m g L^{-1}$ & $K_{b_{1}}$ & 1,000 & $b_{2}(x, 0)$ & $00,2 \mathrm{mgL}^{-1}$ \\
& & $K_{b_{2}}$ & 1,000 & $\frac{\partial C_{i}(L, t)}{\partial x}, i=1,2,3$ & $00.0 m g L^{-1}$ \\
\hline
\end{tabular}

${ }^{*}$ Condição Inicial e Contorno.

A Figura 5 mostra dois gráficos que simulam o comportamento deste sistema com e sem o fator de inibição não competitivo $I_{n}$ em 68 dias. Nota-se que com este fator, ou seja, quando se considera que a presença do aceptor 1 inibe o aceptor 2, a biomassa 2 atua na degradação do doador apenas na região da pluma onde o aceptor 1 já foi consumido ou encontra-se em concentração suficientemente baixa. A taxa de crescimento bacteriano anaeróbio $\left(b_{2}\right)$ nesta região não é acentuada, mas consegue reduzir a quantidade de doador contaminante em uma zona de atividade biológica que chega quase na metade da região de interesse. Quando não há inibição os dois mecanismos, aeróbio e anaeróbio, atuam simultaneamente, reduzindo a concentração da pluma contaminante ao nível obtido na situação com o fator de inibição, porém muito mais rápido e numa zona de atividade biológica bem próximo à fonte de contaminação. A situação com fator de inibição é mais realista visto que o mecanismo anaeróbio é muito mais lento que o metabolismo aeróbio. 

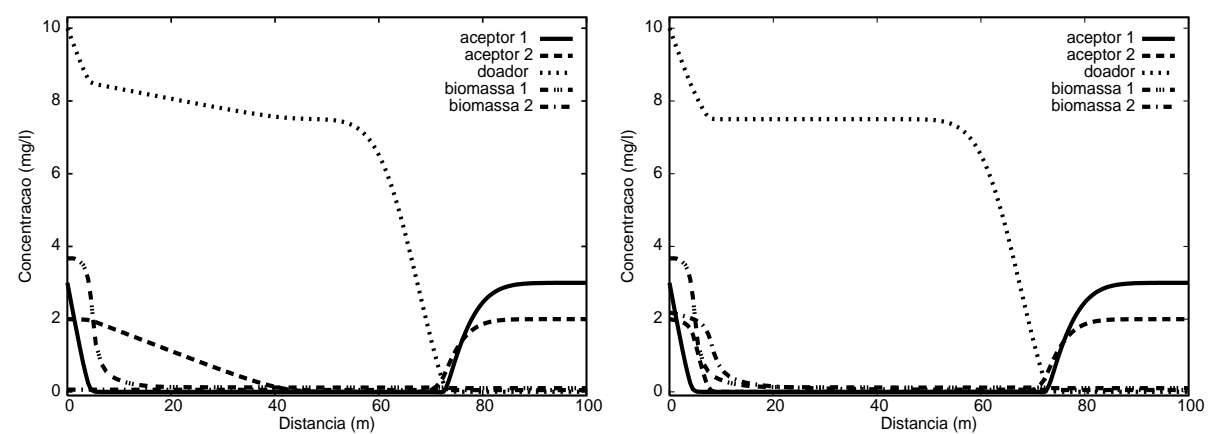

Figura 5: Perfis de concentração em 68 dias para o doador de elétrons (linha pontilhada), o aceptor 1 (linha contínua), o aceptor 2 (linha tracejada), a biomassa 1 (linha traço-3 pontos) e a biomassa 2 (linha traço-ponto) com (esquerda) e sem (direita) inibição não competitiva.

\section{Comentários Finais}

Foram analisados neste trabalho problemas de transporte de contaminantes reativos não-lineres considerando-se processos de biodegradação aeróbios e anaeróbios e proposta uma metodologia numérica CA para solução destes problemas. Esta metodologia aproximou as soluções sem resolver separadamente os operadores diferenciais de cada equação, evitando assim a introdução de possíveis erros de decomposição. Além disso, foram usadas técnicas numéricas reconhecidamente adequadas ao comportamneto físico de todos os fenômenos considerados: Euler Implícito, que é de primeira ordem e incondicionalmente estável, na aproximação das derivadas temporais, SUPG, que adiciona de forma consistente estabilidade em problemas fortemente advectivos e um algoritmo baseado no método de Newton para tratar as não-linearidades e os acoplamentos introduzidos pelo processo reativo de biodegradação. Analisou-se dois cenários de contaminação possíveis de acontecer na sub-superfície, facilitando assim o entendimento dos fenômenos envolvidos nos processos de biodegradação durante o transporte de contaminantes. O primeiro deles demonstrou a importância de se ter níveis suficientes de aceptores de elétrons na subsuperfície para que biodegradação aeróbia seja um processo de remediação eficaz. O segundo destacou que biodegradação aeróbia e anaeróbia podem ocorrer juntas numa pluma contaminada, mas o processo aeróbio pode inibir o anaeróbio e revelar níveis maiores de contaminação ao redor da fonte. A metodologia introduzida e o modelo geral apresentado podem ser facilmente adaptados para incluir processos de sorção, tornando assim o problema ainda mais realista [5].

\footnotetext{
Abstract. We proposed two mathematical models for the nonlinear reactive contaminant transport in the subsurface. First we consider the transport with aerobic biodegradation of two contaminants and then we study the transport under aerobic and anaerobic biodegradation processes in the presence of one pollutant species. A fully coupled numerical methodology (CA) is presented to numerically solve the models. It employs the SUPG (Streamline Upwind Petrov Galerkin) finite elements method and the Implicit Euler finite differences method in space and time
} 
discretization, respectively. Besides, an iterative algorithm associated to the Newton method is applied to linearized and uncouple the resulting equation systems. Numerical simulations are exhibited to demonstrate and illustrate the efficiency of the proposed methodologies when applied to groundwater contamination scenarios.

\section{Referências}

[1] D.A. Barry, H. Prommer, C.T. Miller, P. Engesgaard, A. Brun, C. Zheng, Modelling the fate of oxidisable organic contaminants in groundwater, Advances in Water Resources, 25 (2002), 945-983.

[2] L.S.J. Bell, P.J. Binning, A split operator approach to reactive transport with the forward particle tracking Eulerian Lagrangian localized adjoint method, Advances in Water Resources, 27 (2004), 323-334.

[3] A.N. Brooks, T.J.R. Hughes, Streamline upwind Petrov-Galerkin formulations for convection dominated flows with particular emphasis on compressible Navier-Stokes equations, Comput. Methods Appl. Mech. Engrg., 32, No. 3 (1982), 199-259.

[4] D.K. Button, Kinetics of Nutrient-Limited Transport and Microbial Growth, Microbiological Reviews, 49, No. 3 (1985), 270-297.

[5] P.R.L. Couto, "Modelagem Computacional do Transporte de Contaminantes com Processos de Biodegradação e Sorção Física em um Meio Poroso Saturado", Tese de Doutorado, Laboratório Nacional de Computação Científica, Petrópolis, RJ, 2006.

[6] M.C.C. Cunha, "Métodos Numéricos", Editora da UNICAMP, Campinas, 2003.

[7] C. Johnson, U. Nävert, J. Pitikäranta, Finite element for hyperbolic problemas, Comput. Methds. Appl. Mech. Engrg., 54 (1984), 285-312.

[8] J.S. Kindred, M.A. Celia, Contaminant transport and biodegradation 2. Conceptual model and test simulations, Water Resources Research, 25, No. 6 (1989), 1149-1159.

[9] W.H. Press, S.A. Teukolsky, W.T. Vetterling, B.P. Flannery, "Numerical Recipes in FORTRAN, The Art of Scientific Computing", Cambridge University Press, Cambridge, 1992. 